\title{
Article \\ Sub-Inhibitory Concentrations of Ciprofloxacin Alone and Combinations with Plant-Derived Compounds against $P$. aeruginosa Biofilms and Their Effects on the Metabolomic Profile of $P$. aeruginosa Biofilms
}

\author{
Didem Kart ${ }^{1, * \mathbb{C}}$, Tuba Reçber ${ }^{2}$, Emirhan Nemutlu ${ }^{2,3}$ and Meral Sagiroglu ${ }^{1}$ \\ 1 Department of Pharmaceutical Microbiology, Faculty of Pharmacy, Hacettepe University, \\ Ankara 06100, Turkey; mozalp@hacettepe.edu.tr \\ 2 Department of Analytical Chemistry, Faculty of Pharmacy, Hacettepe University, Ankara 06100, Turkey; \\ tuba.recber@hacettepe.edu.tr (T.R.); enemutlu@hacettepe.edu.tr (E.N.) \\ 3 Bioanalytic and Omics Laboratory, Faculty of Pharmacy, Hacettepe University, Ankara 06100, Turkey \\ * Correspondence: dturk@hacettepe.edu.tr; Tel.: +90-533-690-7637
}

Citation: Kart, D.; Reçber, T.;

Nemutlu, E.; Sagiroglu, M.

Sub-Inhibitory Concentrations of

Ciprofloxacin Alone and

Combinations with Plant-Derived

Compounds against $P$. aeruginosa

Biofilms and Their Effects on the

Metabolomic Profile of $P$. aeruginosa

Biofilms. Antibiotics 2021, 10, 414.

https: / / doi.org/10.3390/

antibiotics 10040414

Academic Editor: Eliana De Gregorio

Received: 2 March 2021

Accepted: 6 April 2021

Published: 9 April 2021

Publisher's Note: MDPI stays neutral with regard to jurisdictional claims in published maps and institutional affiliations.

Copyright: (c) 2021 by the authors. Licensee MDPI, Basel, Switzerland. This article is an open access article distributed under the terms and conditions of the Creative Commons Attribution (CC BY) license (https:// creativecommons.org/licenses/by/ $4.0 /)$.

\begin{abstract}
Introduction: Alternative anti-biofilm agents are needed to combat Pseudomonas aeruginosa infections. The mechanisms behind these new agents also need to be revealed at a molecular level. Materials and methods: The anti-biofilm effects of 10 plant-derived compounds on P. aeruginosa biofilms were investigated using minimum biofilm eradication concentration (MBEC) and virulence assays. The effects of ciprofloxacin and compound combinations on P. aeruginosa in mono and triple biofilms were compared. A metabolomic approach and qRT-PCR were applied to the biofilms treated with ciprofloxacin in combination with baicalein, esculin hydrate, curcumin, and cinnamaldehyde at sub-minimal biofilm inhibitory concentration (MBIC) concentrations to highlight the specific metabolic shifts between the biofilms and to determine the quorum sensing gene expressions, respectively. Results: The combinations of ciprofloxacin with curcumin, baicalein, esculetin, and cinnamaldehyde showed more reduced MBICs than ciprofloxacin alone. The quorum sensing genes were downregulated in the presence of curcumin and cinnamaldehyde, while upregulated in the presence of baicalein and esculin hydrate rather than for ciprofloxacin alone. The combinations exhibited different killing effects on P. aeruginosa in mono and triple biofilms without affecting its virulence. The findings of the decreased metabolite levels related to pyrimidine and lipopolysaccharide synthesis and to down-regulated alginate and lasI expressions strongly indicate the role of multifactorial mechanisms for curcumin-mediated P. aeruginosa growth inhibition. Conclusions: The use of curcumin, baicalein, esculetin, and cinnamaldehyde with ciprofloxacin will help fight against P. aeruginosa biofilms. To the best of our knowledge, this is the first study of its kind to define the effect of plant-based compounds as possible anti-biofilm agents with low MBICs for the treatment of P. aeruginosa biofilms through metabolomic pathways.
\end{abstract}

Keywords: P. aeruginosa; plant-derived compounds; metabolomic; MBIC; biofilm

\section{Introduction}

Biofilm-related infections have major importance because of their clinical challenges, such as persistence and treatment approach. Pseudomonas aeruginosa, which is difficult to treat because of its resistance to many antibiotics and its ability to form a biofilm, is a significant pathogen that causes hospital-acquired infections [1]. P. aeruginosa is frequently isolated together with Staphylococcus aureus and Enterococcus faecalis from the specimens of patients with biofilm-related infections [2]. There is an urgent need to develop new antimicrobial approaches that prevent or inhibit $P$. aeruginosa biofilm formation or display a microbicidal activity on the cells in preformed biofilms [3]. Quorum sensing is a communication system in which microorganisms control their population density through 
small signal molecules [4]. Because of the importance of QS controlled ability for the formation and/or dispersion of P. aeruginosa biofilms, QS inhibitors are recommended as alternative anti-biofilm agents to antibiotics [5]. QS inhibitors can limit the development of drug resistance by applying selective pressure at concentrations below the minimum inhibitory concentration (MIC) [6]. Because of its non-toxic nature, the effect of various natural compounds on P. aeruginosa biofilms has been studied. Although the inhibitory effects of some natural compounds on the P. aeruginosa QS system are known, their effects on $P$. aeruginosa cells in polymicrobial biofilm media are still under investigation $[7,8]$. Investigating the effects of these natural compounds on biofilm cells with genomic and metabolomic analyzes will be effective for elucidating their anti-biofilm mechanisms.

The present study determined the minimal biofilm inhibitory concentration (MBIC) and minimum biofilm eradication concentration (MBEC) of ciprofloxacin in combination with plant-derived compounds such as curcumin, azathioprine, resveratrol, catechin hydrate, baicalin hydrate, L-canavanine, 4-nitropyridine N-oxide, p-benzoquinone, esculin hydrate, and cinnamaldehyde against $P$. aeruginosa biofilm cells. The effect of sub-MBIC concentrations of curcumin, baicalein, cinnamaldehyde, and esculin hydrate, which show the best synergistic activity with ciprofloxacin, on the QS genes and virulence characteristics of $P$. aeruginosa was investigated. To observe the antimicrobial effects of these compounds and the ciprofloxacin combinations on polymicrobial biofilms at sub-MBIC concentrations, $P$. aeruginosa cells in mono- and triple-species biofilms were separately treated with them and the results were compared. A GC-MS based metabolomic approach was used to reveal the underlying mechanisms of the anti-biofilm and anti-QS activity of curcumin, baicalein, cinnamaldehyde, and esculin hydrate.

\section{Materials and Methods}

\subsection{Bacterial Strains and Growth Conditions}

P. aeruginosa ATCC 47085 (PAO1), E. faecalis ATCC 47077, and S. aureus ATCC 35556 were used in the study. Fresh cultures were grown on brain heart infusion agar (BHIA; Oxoid Ltd., Hampshire, UK) at $37^{\circ} \mathrm{C}$ for $24 \mathrm{~h}$. The overnight cultures were prepared by subculturing bacterial strains in brain heart infusion broth (BHI; Oxoid Ltd., Hampshire, UK) and incubated at $37^{\circ} \mathrm{C}$ for $24 \mathrm{~h}$. For all of the experiments, the final bacterial suspensions were diluted with $\mathrm{BHI}$ to $10^{6}$ colony forming units per $\mathrm{mL}(\mathrm{cfu} / \mathrm{mL})$.

\subsection{Antibiotics and Plant-Derived Compounds}

Curcumin, azathioprine, resveratrol, catechin hydrate, baicalin hydrate, L-canavanine, 4nitropyridine $\mathrm{N}$-oxide, p-benzoquinone, esculin hydrate, cinnamaldehyde, and ciprofloxacin were purchased from Sigma Chemical Co. (St. Louis, MO, USA). Stock solutions of ciprofloxacin and the compounds were prepared in dimethyl sulfoxide (pure DMSO), sterile water, or hydrochloric acid according to the manufacturer's recommendations.

\subsection{MIC, MBIC, and MBEC Assay}

The MICs of ciprofloxacin and the compounds were determined using the broth microdilution method according to Clinical Laboratory Standards Institute (CLSI) guidelines [9]. P. aeruginosa ATCC 27853 was used as quality control for this test.

MBEC Assay ${ }^{\mathrm{TM}}$ (Physiology and Genetics, P\&G, Innovotech Inc., Edmonton, AB, Canada) was used to evaluate the anti-biofilm activity of the agents against $P$. aeruginosa PAO1 biofilms following the test protocol provided by the manufacturer. Briefly, $150 \mu \mathrm{L}$ of $P$. aeruginosa suspension containing $10^{6} \mathrm{cfu} / \mathrm{mL}$ was transferred to each test well and the MBEC assay plate lids with 96 pegs were placed in the microtiter plate wells to form $24 \mathrm{~h}$ mature biofilms at $37^{\circ} \mathrm{C}$. After washing, the peg lids were placed into the challenge plate wells, which contained a serial two-fold dilution of each agent alone and their combinations for $24 \mathrm{~h}$ of incubation. In columns 1 to 11 , the tested concentration ranges of ciprofloxacin and the compounds were $1-0.001 \mathrm{mg} / \mathrm{L}$ and $8.2-0.008 \mathrm{mg} / \mathrm{L}$, respectively. After the MBIC of ciprofloxacin was determined to be $0.128 \mathrm{mg} / \mathrm{L}$, the test concentration ranges 
for use in combination with the compounds were determined as $0.128-0.00128 \mathrm{mg} / \mathrm{L}$ between columns 1 to 11 . Following incubation, the sonication steps were performed to transfer the biofilms on the pegs to the recovery medium, followed by overnight incubation. The optical density of each well was measured at $550 \mathrm{~nm}$. MBEC is defined as the lowest concentration of a compound that prevents the regrowth of bacteria from treated-biofilms and MBIC is the minimum concentration that inhibits bio-film formation. Sub-MBIC values corresponding to $1 / 2 \times$ MBIC of combinations of ciprofloxacin with compounds were used in the subsequent biofilm experiments, including the virulence, gene expressions, and metabolomic analyzes.

2.4. Evaluation of the Antibiofilm Effects of Ciprofloxacin in Combination with the Compounds on Mono- and Triple-Species Biofilms of P. aeruginosa PAO1 and SEM Analysis

P. aeruginosa ATCC 47085 (PAO1) biofilms and triple-species biofilms formed by P. aeruginosa PAO1, E. faecalis ATCC 47077, and S. aureus ATCC 35556 were formed in 96-well microtiter plates as described by Kart et al. [10]. For triple-species biofilms, the starting inoculum of E. faecalis and S. aureus in equal volume with P. aeruginosa was used.

Ciprofloxacin alone and the combination of ciprofloxacin with compounds at subMBIC $(1 / 2 \times$ MBIC) concentrations were applied on the mature mono- and triple-species biofilms. For the quantification of $P$. aeruginosa in the mono- and triple-species biofilms, the plating was performed on BHIA and Cetrimide agar, respectively. After $24 \mathrm{~h}$, the colonies were counted as cfu/mL. All of the experiments were carried out at least three times.

SEM analyses were performed to visualize mono- and triple-species biofilms (Supplement Figure S1). Briefly, final suspensions of P. aeruginosa, S. aureus, and E. faecalis strains containing $\sim 10^{6} \mathrm{CFU} / \mathrm{mL}$ in BHI were added alone (for P. aeruginosa) and in combination $(1: 1, \mathrm{vol} / \mathrm{vol})$ onto the coverslips and were incubated at $37^{\circ} \mathrm{C}$ for $24 \mathrm{~h}$. Following the incubation time, the coverslips were washed twice with phosphate-buffered saline and fixed in a buffer containing $2 \%$ glutaraldehyde and $0.1 \mathrm{M}$ cacodylate for $30 \mathrm{~min}$, followed by rinsing three times for $10 \mathrm{~min}$ in a $0.2 \mathrm{M}$ cacodylate buffer. After passing them through serial ethanol solutions, the samples were dried and then coated with gold-palladium [11].

\subsection{Alterations in Virulence Characteristics of P. aeruginosa Biofilm Cells}

\subsubsection{Swarming}

Ciprofloxacin $(0.0005,0.001$, and $0.004 \mathrm{mg} / \mathrm{L})$, compounds (the same sub-MBIC values in the combinations) and ciprofloxacin-compound combinations $(1 / 2 \times \mathrm{MBIC})$ were added to nutrient agar $(8 \mathrm{~g} / \mathrm{L})$ supplemented with $5 \%$ glucose. The plates were then inoculated with $2 \mu \mathrm{L}$ of diluted P. aeruginosa biofilm cultures and incubated for $16 \mathrm{~h}$ at $37^{\circ} \mathrm{C}$. The zone diameter on the surfaces was measured to assess the swarming activity.

\subsubsection{Twitching}

Luria-Bertani agar plates were prepared to contain ciprofloxacin $(0.0005,0.001$, and $0.004 \mathrm{mg} / \mathrm{L}$ ), compounds (the same sub-MBIC values in the combinations), and ciprofloxacincompound combinations $(1 / 2 \times$ MBIC). The biofilm cultures of $P$. aeruginosa were stabbed from the agar surface to the bottom of the plate with a sterile needle loop. The plates were then incubated at $37^{\circ} \mathrm{C}$ for $48 \mathrm{~h}$. After the agar was removed, the polystyrene surface of the plates was gently washed and stained with crystal violet $(0.1 \%, w / v)$. The twitching motility was assessed by measuring the diameter of the stained zone, indicating that bacteria were attached to the polystyrene surface.

\subsubsection{Quantification of Pyocyanin}

Briefly, $1 \mathrm{~mL}$ of the treated $(1 / 2 \times$ MBIC) and untreated P. aeruginosa biofilm cultures was extracted with $1 \mathrm{~mL}$ of chloroform and then re-extracted into $1 \mathrm{~mL}$ of $0.2 \mathrm{~N} \mathrm{HCl}$ to give a pink solution. The pyocyanin absorbance of this acidic solution was measured at $520 \mathrm{~nm}$ [12]. The results are expressed as \% reduction in pyocyanin in treated biofilm cultures compared with untreated biofilm cultures. 


\subsection{Expression of the QS-Related Genes in Biofilms}

The total RNAs in biofilm cells treated with sub-MBICs $(0.0005$ and $0.001 \mathrm{mg} / \mathrm{L})$ of ciprofloxacin alone and ciprofloxacin $(0.0005$ and $0.001 \mathrm{mg} / \mathrm{L})$ in combination with curcumin $(0.004 \mathrm{mg} / \mathrm{L})$, baicalein $(0.004 \mathrm{mg} / \mathrm{L})$, cinnamaldehyde $(0.008 \mathrm{mg} / \mathrm{L})$, and esculetin hydrate $(0.008 \mathrm{mg} / \mathrm{L})$ were obtained by RNA Isolation Kit (Roche Life Science) according to the manufacturer's instructions. The measurements of the mRNA levels of the QS-related genes were carried out based on our previous reference study [13]. The primers used are listed in Supplementary Table S2.

\subsection{Metabolomic Analysis}

Metabolites were extracted from the biofilm cells treated with ciprofloxacin $(0.0005$ and $0.001 \mathrm{mg} / \mathrm{L})$ alone and ciprofloxacin $(0.0005$ and $0.001 \mathrm{mg} / \mathrm{L})$ in combination with curcumin $(0.004 \mathrm{mg} / \mathrm{L})$, baicalein $(0.004 \mathrm{mg} / \mathrm{L})$, cinnamaldehyde $(0.008 \mathrm{mg} / \mathrm{L})$, and esculetin hydrate $(0.008 \mathrm{mg} / \mathrm{L})$. GC-MS-based measurement of the metabolites was carried out based on our previous reference study [11] (Table S1).

\subsection{Statistical Analysis}

The colony counts were recorded for each treatment as Log10. All statistical calculations were performed on the log density values. The statistical significance of the data was determined by Student's t-test for paired samples or by the non-parametric Wilcoxon signed-rank test. A $p$-value of $<0.05$ was considered significant.

\section{Results}

\subsection{Effect of the Compounds and Ciprofloxacin on the Planktonic and Biofilm Cells of P. aeruginosa}

Based on the broth microdilution method, the MIC values of all compounds and antibiotics against $P$. aeruginosa are shown in Table 1 . When ciprofloxacin and the compounds were administered both alone and in combination, the MBIC and MBEC values obtained against the $P$. aeruginosa biofilm cells were determined by the MBEC test. The compounds alone $(8.2-0.004 \mathrm{mg} / \mathrm{L})$ did not affect destroying cells in either biofilm. However, in the presence of the compounds, it was observed that the MBICs of ciprofloxacin were much lower compared with the MBIC of ciprofloxacin alone (Table 1). Of all the compounds in the combinations, curcumin, baicalein, esculetin hydrate, and cinnamaldehyde were the most effective ones at reducing the MBICs of ciprofloxacin. No significant difference was observed in MBEC values when ciprofloxacin was administered alone or in combination with compounds (Table 1).

Table 1. MICs, MBICs, and MBECs of the compounds and ciprofloxacin against P. aeruginosa PAO1.

\begin{tabular}{|c|c|c|c|c|c|}
\hline \multirow[b]{2}{*}{ Agents } & \multirow{2}{*}{$\begin{array}{c}\text { MICs (mg/L) } \\
\text { Alone * }\end{array}$} & \multicolumn{2}{|c|}{ MBICs (mg/L) } & \multicolumn{2}{|c|}{ MBECs (mg/L) } \\
\hline & & Alone ** & $\begin{array}{l}\text { Combinations } \\
\text { (CIP + Compounds) }\end{array}$ & Alone $* *$ & $\begin{array}{l}\text { Combinations } * * * \\
\text { (CIP + Compounds) }\end{array}$ \\
\hline Ciprofloxacin & 0.039 & 0.06 & - & 0.13 & - \\
\hline Curcumin & 0.25 & 0.82 & $0.001+0.008$ & $>0.82$ & $0.13+2$ \\
\hline Azathioprine & 0.25 & 0.82 & $0.008+0.06$ & $>0.82$ & $0.13+2$ \\
\hline Resveratrol & 0.25 & 0.2 & $0.03+0.25$ & $>0.82$ & $0.13+2$ \\
\hline Catechin hydrate & 0.51 & 0.82 & $0.03+0.26$ & $>0.82$ & $>0.13+2$ \\
\hline Baicalein & 0.13 & 0.2 & $0.001+0.008$ & $>0.82$ & $0.13+2$ \\
\hline L-canavanine & 0.1 & 0.82 & $0.004+0.03$ & $>0.82$ & $0.13+2$ \\
\hline 4-nitropyridine $\mathrm{N}$-oxide & 0.02 & 0.41 & $0.008+0.06$ & $>0.82$ & $0.13+2$ \\
\hline p-benzoquinone & 0.06 & 0.41 & $0.008+0.06$ & $>0.82$ & $0.13+2$ \\
\hline Esculetin hydrate & 0.51 & 0.82 & $0.002+0.02$ & $>0.82$ & $0.13+2$ \\
\hline Cinnamaldehyde & 0.26 & 0.41 & $0.002+0.02$ & $>0.82$ & $0.13+2$ \\
\hline
\end{tabular}

MICs-minimum inhibitory concentrations; MBICs-minimum biofilm inhibitory concentrations; MBECs-minimum biofilm eradication concentrations; $\mathrm{mg} / \mathrm{L}-$ milligram per liter. ${ }^{*}$ Minimum inhibition concentrations at which antimicrobial agents alone act against $P$. aeruginosa planktonic cells. ${ }^{* *}$ Minimum biofilm inhibition concentrations at which antimicrobial agents alone act against $P$. aeruginosa biofilm cells.

${ }_{* * *}$ Minimum biofilm eradication concentrations at which compounds combined with ciprofloxacin act against $P$. aeruginosa biofilm cells. 
3.2. Comparison of the Effect of Compound-Ciprofloxacin Combinations with Sub-MBIC Values on Mono- and Triple-Species Biofilms of P. aeruginosa

Mono- and triple-species biofilm models have been developed to understand whether the efficacy of antimicrobial combinations at sub-MBIC concentrations differ when P. aeruginosa is in a mixed biofilm environment compared with alone. When ciprofloxacin was combined with azathioprine, an approximately 6 logarithm further reduction in the number of $P$. aeruginosa viable cells in the mono-species biofilms than that of triple-species biofilm was observed. In contrast, the viable cell counts in mono-species biofilms treated with the combination of ciprofloxacin with curcumin, catechin hydrate, baicalein, L-canavanine, esculin hydrate, and cinnamaldehyde were found to be less than the triple-species biofilms (Figure 1). No significant difference was observed in the cell numbers treated with the combination of ciprofloxacin with resveratrol, 4-nitropyridine $\mathrm{N}$-oxide, and p-benzoquinone in both biofilm models (Figure 1). According to our results, combinations of antimicrobial agents may show different effects in mono- or triple-species biofilms.

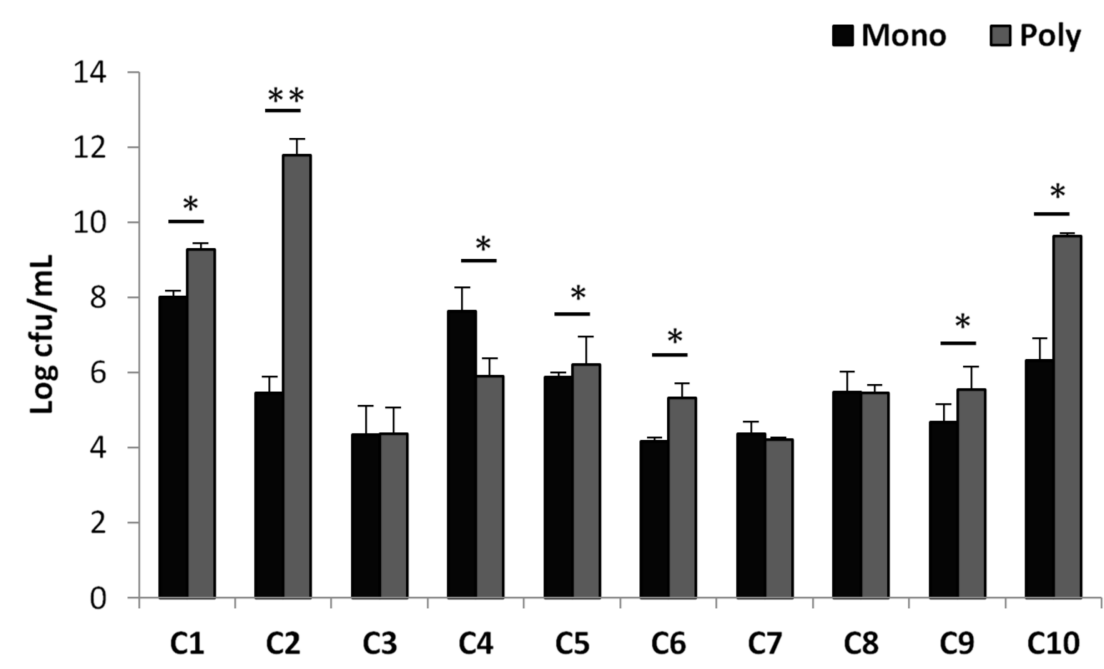

Figure 1. Viable cell counts of P. aeruginosa in mono- and triple-species biofilms. C1: Ciprofloxacin + Curcumin; C2: Ciprofloxacin + Azathioprine; C3: Ciprofloxacin + Resveratrol; C4: Ciprofloxacin + Catechin hydrate, C5: Ciprofloxacin + Baicalein, C6: Ciprofloxacin + L-canavanine, C7: Ciprofloxacin + 4-nitropyridine N-oxide; C8: Ciprofloxacin + p-benzoquinone; C9: Ciprofloxacin + Esculetin hydrate; C0: Ciprofloxacin + Cinnamaldehyde. ${ }^{*}$ Significant $(p<0.05),{ }^{* *}$ Significant $(p<0.01)$. The results are shown as the average \pm standard deviation.

\subsection{Influence of the Compounds and Ciprofloxacin Combinations on Virulence Properties of $P$. aeruginosa}

Compared with the untreated $P$. aeruginosa biofilms, treatment with cinnamaldehyde and p-benzoquinone caused a significant inhibition in the swarming motility of bacteria, while treatment with azathioprine resulted in a significant increase in the cells motility (Figure 2A). No inhibition of swarming motility was observed in biofilm cells treated with curcumin, baicalein, and esculin hydrate compared with the control. Combinations of all compounds tested with ciprofloxacin exhibited an inhibitory effect similar to that of ciprofloxacin alone compared with untreated biofilm cultures. In addition, no additional contribution of the combinations to ciprofloxacin in inhibiting Pseudomonas swarming activity was observed (Figure 2A). 
A

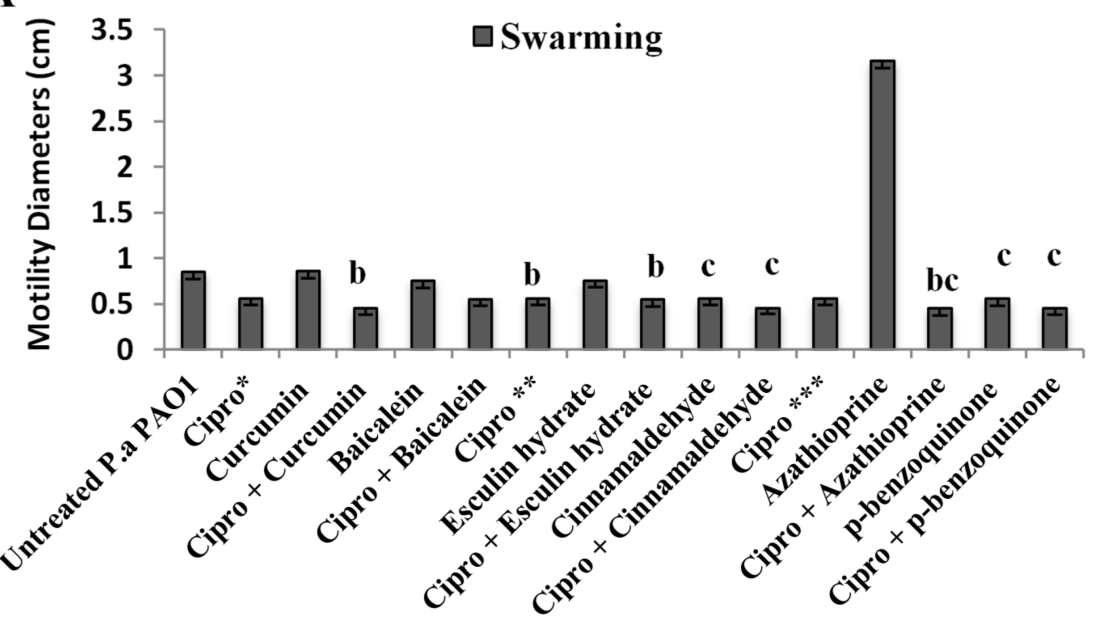

B

$\square$ Twitching
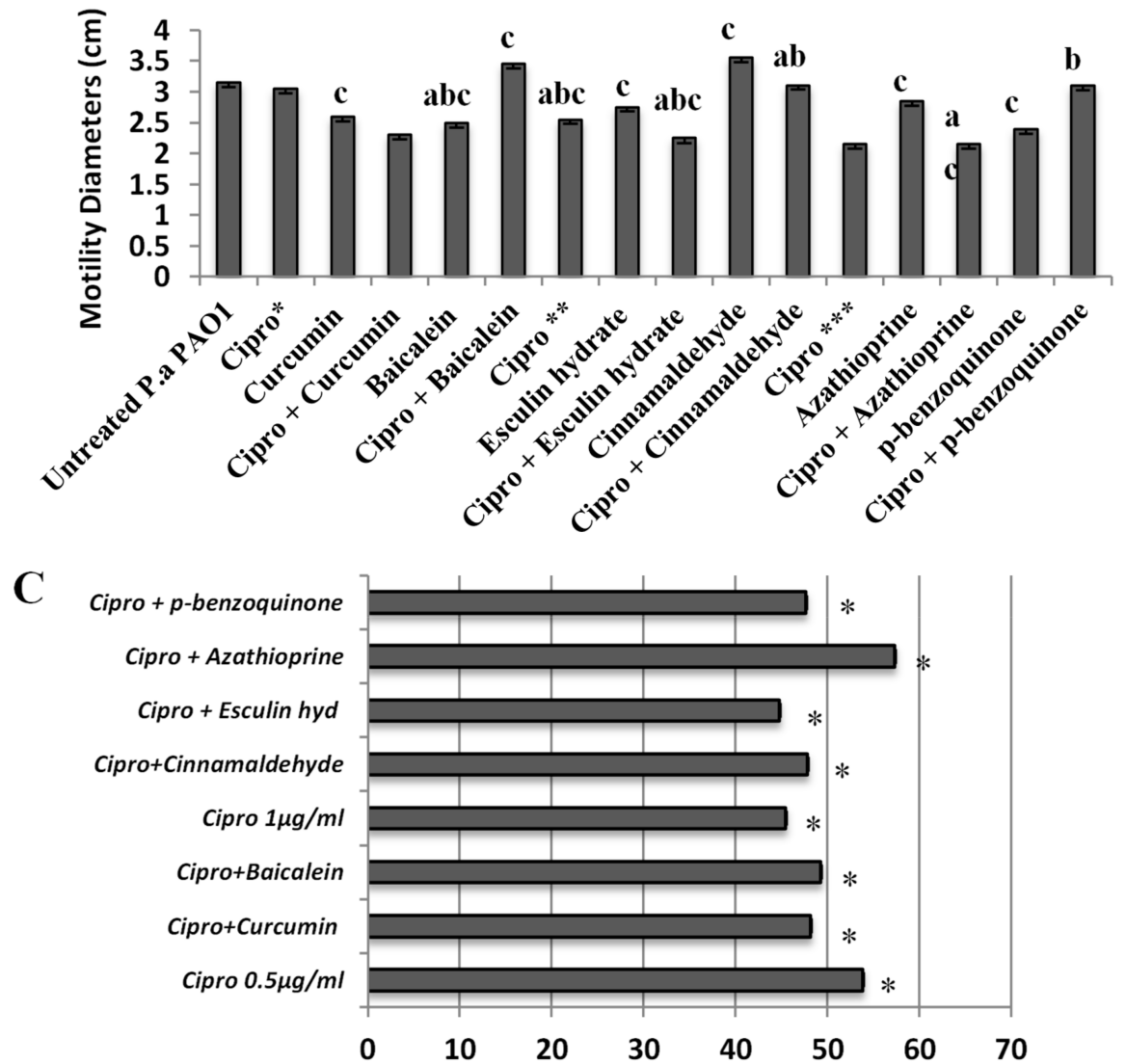

Figure 2. The effect of the antimicrobial agents alone and in combination on the P. aeruginosa biofilm cells: (A) twitching motilities and (B) swarming motilities. The measurements of the swarming and twitching activity (in centimeter) were performed on the agar plates inoculated with the cells detached from biofilms treated with antimicrobial agents. Cipro*: Ciprofloxacin at $0.0005 \mathrm{mg} / \mathrm{L}$; Cipro**: Ciprofloxacin at $0.001 \mathrm{mg} / \mathrm{L} ; \mathrm{Cipro}^{* * *}$ : Ciprofloxacin at $0.004 \mathrm{mg} / \mathrm{L}$. (a) It is significantly different than the cells treated with ciprofloxacin $(p<0.05)$. (b) It is significantly different than the cells treated with the compounds $(p<0.05)$. (c) It is significantly different than the untreated cells $(p<0.05)$. (C) Quantitation of pyocyanin production in the biofilm cells treated with ciprofloxacin alone and in combination with the compounds. * Significant $(p<0.05)$. The results are expressed as \% reduction. 
For the twitching activity, ciprofloxacin and all compounds except cinnamaldehyde caused statistically significant inhibition compared with the untreated control. The twitching activity of $P$. aeruginosa was inhibited after treatment with ciprofloxacin and all compounds tested separately, while in combination, only ciprofloxacin with the curcumin and esculetin hydrate compounds were effective in inhibiting the twitching motility of the cells. However, when baicalein was combined with ciprofloxacin, the motility of the cells was increased (Figure 2B).

\subsection{The Impact of Ciprofloxacin Combined with the Compounds on QS Gene Expressions of P. aeruginosa Biofilm Cells}

In cells treated with both ciprofloxacin alone and ciprofloxacin in combination with curcumin and cinnamaldehyde on the expression levels of lasl, alg, and psl genes related to QS and biofilm formation were decreased. On the contrary, increased expressions were observed in cells treated with ciprofloxacin in combination with baicalein and esculin hydrate (Figure 3).
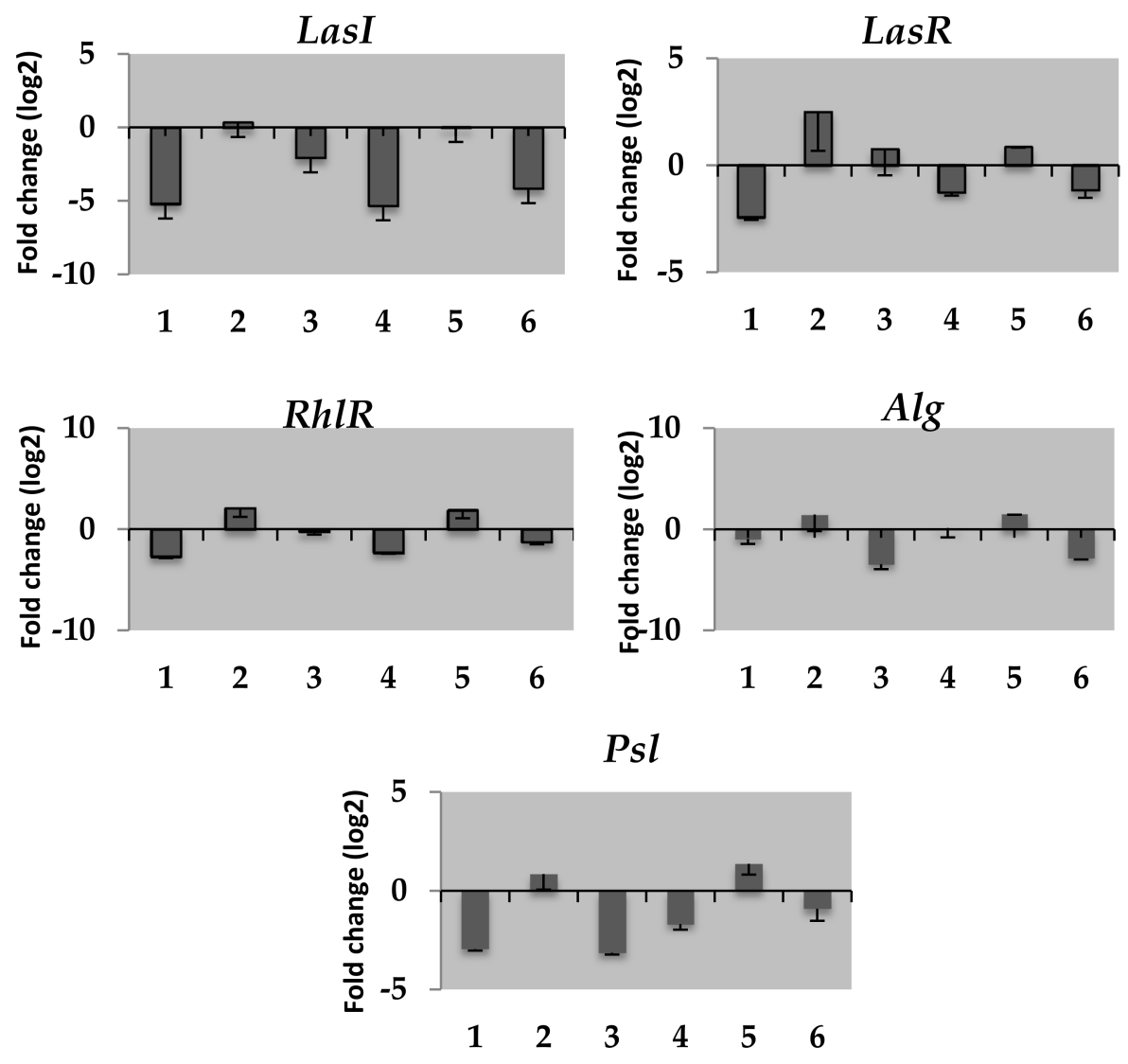

Figure 3. The mean relative mRNA expression levels of quorum sensing genes in P. aeruginosa cells treated with ciprofloxacin alone and in combination with curcumin, cinnamaldehyde, baicalein and esculetin hydrate. ${ }^{*}$ Indicates statistically significant $(p<0.05) 1$. Ciprofloxacin $(0.5 \mu \mathrm{g} / \mathrm{mL})$, 2. Ciprofloxacin $(0.5 \mu \mathrm{g} / \mathrm{mL})$ and Baicalein $(4 \mu \mathrm{g} / \mathrm{mL}), 3$. Ciprofloxacin $(0.5 \mu \mathrm{g} / \mathrm{mL})$ and Curcumin $(4 \mu \mathrm{g} / \mathrm{mL})$, 4. Ciprofloxacin $(1 \mu \mathrm{g} / \mathrm{mL}), 5$. Ciprofloxacin $(1 \mu \mathrm{g} / \mathrm{mL})$ and Esculetin hydrate $(8 \mu \mathrm{g} / \mathrm{mL})$, 6. Ciprofloxacin $(1 \mu \mathrm{g} / \mathrm{mL})+$ Cinnamaldehyde $(8 \mu \mathrm{g} / \mathrm{mL})$.

3.5. Effect of Ciprofloxacin and Compounds Combinations on the Metabolic Profile of P. aeruginosa Biofilm Cells

After treatment with both sub-MBICs of ciprofloxacin and combinations of ciprofloxacin with curcumin, baicalein, cinnamaldehyde, and esculetin hydrate, the changed metabolite profiles in the biofilm cells are shown in Table 2 and Figure 4. Many metabolic pathways such as carbohydrates, amino acids, nitrogen, amines, lipids, and nucleotides were re- 
duced following the addition of curcumin compared with ciprofloxacin alone. Compared with ciprofloxacin alone, statistically significant changes in the metabolites involved in the carbohydrates, amines, and nucleotide pathways were observed in the cells treated with ciprofloxacin-baicalein and ciprofloxacin-esculetin hydrate combinations (Table 3). Compared with baicalein and esculetin hydrate, cinnamaldehyde had different metabolite effects on the amine and pyrimidine pathways. Decreased uracil, uridine monophosphate (UMP), and ribulose-5-phosphate levels were observed in the cells treated with combinations of ciprofloxacin with curcumin and cinnamaldehyde, while increased levels of uracil and UMP were observed in cells treated with combinations of ciprofloxacin with baicalein and esculin hydrate (Tables 2 and 3 ).

Table 2. Comparison of metabolic profiling of P. aeruginosa biofilm cells treated with ciprofloxacin alone and combinations of ciprofloxacin with curcumin and cinnamaldehyde. The biofilm cells were grown in brain heart infusion agar (BHI) broth at $37^{\circ} \mathrm{C}$ for $24 \mathrm{~h}$, followed by further treatment with antimicrobial agents for another $24 \mathrm{~h}$. The results are shown as the average.

\begin{tabular}{|c|c|c|c|c|c|c|c|}
\hline & & Curc. & Cinnam. & & & Curc. & Cinnam. \\
\hline \multirow{9}{*}{ 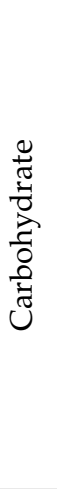 } & Citric acid & $0.44 \downarrow$ & - & \multirow{4}{*}{ 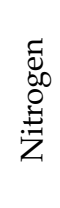 } & Pyroglutamic acid & $0.13 \downarrow$ & $1.7 \uparrow$ \\
\hline & Lactose & $0.32 \downarrow$ & - & & Aspartic acid & $0.03 \downarrow$ & $2.08 \uparrow$ \\
\hline & Glucose & $4.04 \uparrow$ & - & & Glutamic acid & $0.06 \downarrow$ & - \\
\hline & Melibiose & $0.1 \downarrow$ & $1.83 \uparrow$ & & $\mathrm{N}$-acetyl-l-glutamic acid & $0.3 \downarrow$ & - \\
\hline & Mannose & $1.78 \uparrow$ & - & & & & \\
\hline & Maltotriose & $0.02 \downarrow$ & $2.92 \uparrow$ & \multirow{6}{*}{ 尊 } & Glyceric acid & $0.25 \downarrow$ & - \\
\hline & Maltotriitol & $0.01 \downarrow$ & $2.61 \uparrow$ & & Glycine & $0.4 \downarrow$ & - \\
\hline & Melezitose & $0.01 \downarrow$ & $2.3 \uparrow$ & & Glycerol-phosphate & $0.35 \downarrow$ & - \\
\hline & Glucuronic acid & $0.24 \downarrow$ & - & & Beta-glycerolphosphate & $0.42 \downarrow$ & - \\
\hline \multirow{6}{*}{ 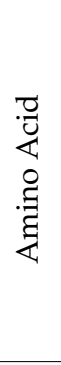 } & Threonine & $0.43 \downarrow$ & - & & Glyceraldehyde & $1.83 \uparrow$ & - \\
\hline & Proline & $0.24 \downarrow$ & - & & 3-phosphoglycerate & $0.04 \downarrow$ & - \\
\hline & Methionine & $0.28 \downarrow$ & - & & & & \\
\hline & Lysine & $0.01 \downarrow$ & $0.12 \downarrow$ & \multirow{6}{*}{ 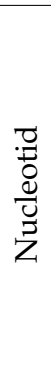 } & Uracil & $0.13 \downarrow$ & $0.17 \downarrow$ \\
\hline & Phenylalanine & $0.49 \downarrow$ & - & & Uridine 5'-monophosphate & $1.5 \uparrow$ & $0.02 \downarrow$ \\
\hline & Tryptophane & & $0.19 \downarrow$ & & Ribulose-5-phosphate & $0.23 \downarrow$ & $0.06 \downarrow$ \\
\hline \multirow{3}{*}{$\underset{\stackrel{\Xi}{Z}}{\stackrel{\Xi}{Z}}$} & Phenylethylamine & $0.03 \downarrow$ & $0.43 \downarrow$ & & Pyrombocnhato & $658 \uparrow$ & 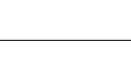 \\
\hline & 2-amino-1-phenylethanol & $0.01 \downarrow$ & $0.42 \downarrow$ & & Cytidine-5'-monophosphate & $0.35 \downarrow$ & - \\
\hline & Tyramine & $0.14 \downarrow$ & - & & Porphine & $3.19 \uparrow$ & - \\
\hline
\end{tabular}



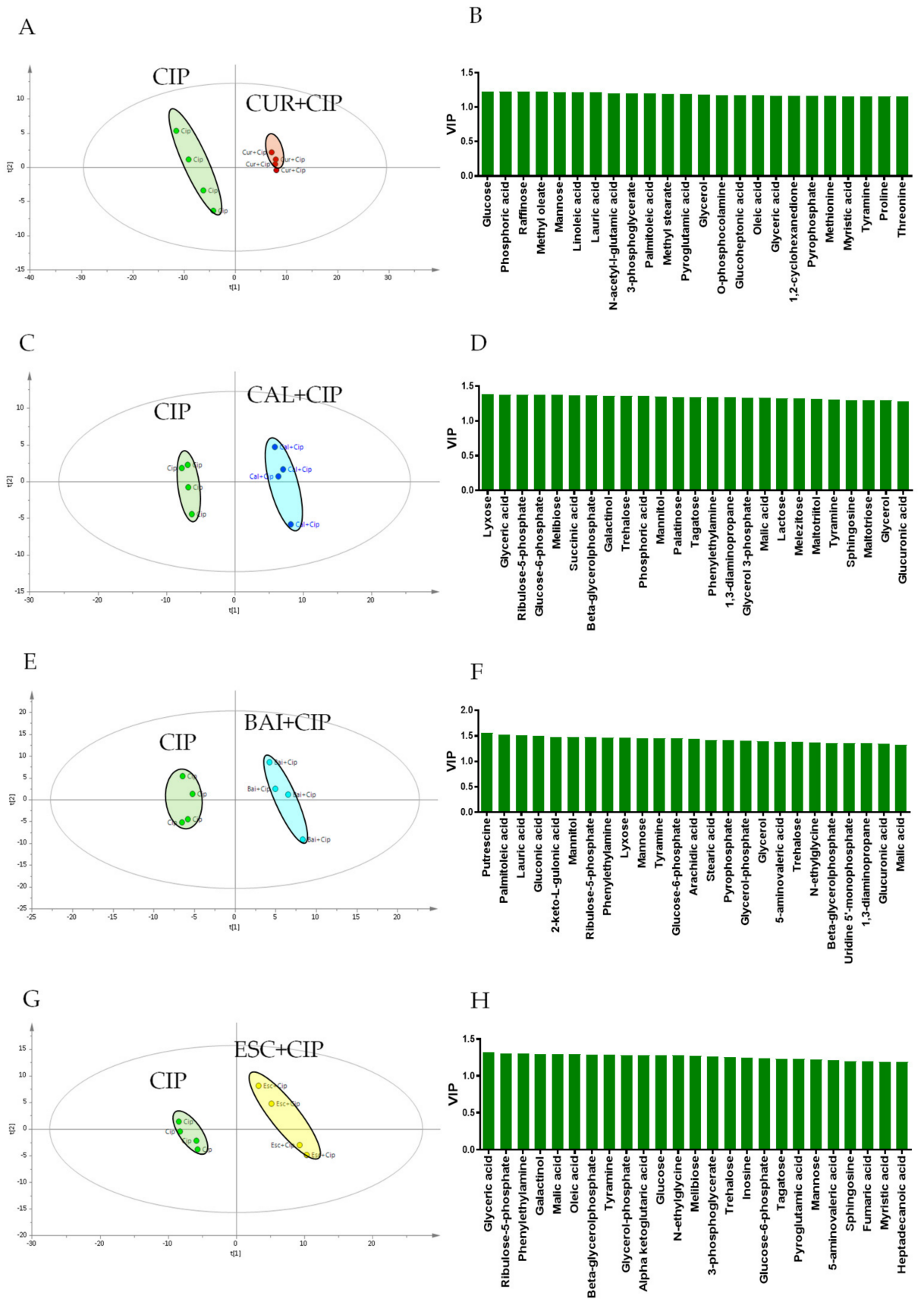

Figure 4. Partial Least-Squares Discriminant Analysis score chart and Variable importance charts obtained after the comparison of biofilm cells treated with ciprofloxacin alone with those treated with combinations of curcumin, cinnamaldehyde, baicalein, and esculetin hydrate. (A) PLS-DA score plot shows a clear separation between $P$. aeruginosa cells treated with ciprofloxacin alone and in combination with curcumin. The R2 and Q2 values for the biofilm samples were 0.999 and 0.983 , respectively. (C) PLS-DA score plot shows a clear separation between P. aeruginosa cells treated with ciprofloxacin alone and in combination with cinnamaldehyde. The R2 and Q2 values for the biofilm samples were 0.998 and 0.971, respectively; (E) PLS-DA score plot shows a clear separation between P. aeruginosa cells treated with ciprofloxacin alone and in combination with baicalein. The R2 and Q2 values for the biofilm samples were 0.995 and 0.960, respectively. (G) PLS-DA score plot shows a clear separation between $P$. aeruginosa cells treated with ciprofloxacin alone and in combination with esculetin hydrate. The R2 and Q2 values for the biofilm samples were 0.998 and 0.990 , respectively. $(\mathbf{B}, \mathbf{D}, \mathbf{F}, \mathbf{H})$ VIP charts of metabolites that are effective in separating the groups. 
Table 3. Comparison of metabolic profiling of P. aeruginosa biofilm cells treated with ciprofloxacin alone and combinations of ciprofloxacin with baicalein and esculetin hydrate. The biofilm cells were grown in $\mathrm{BHI}$ broth at $37^{\circ} \mathrm{C}$ for $24 \mathrm{~h}$, followed by further treatment with antimicrobial agents for another $24 \mathrm{~h}$. The results are shown as the average.

\begin{tabular}{|c|c|c|c|}
\hline & & Esculetin hyd & Baicalein hyd \\
\hline \multirow{8}{*}{ 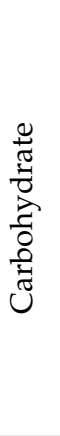 } & Fumaric acid & - & $1.61 \uparrow$ \\
\hline & Malic acid & - & $1.64 \uparrow$ \\
\hline & Melibiose & $1.51 \uparrow$ & $1.71 \uparrow$ \\
\hline & Mannose & $1.47 \uparrow$ & $1.83 \uparrow$ \\
\hline & Maltotriose & $2.08 \uparrow$ & - \\
\hline & Maltotriitol & $1.75 \uparrow$ & - \\
\hline & Melezitose & $1.56 \uparrow$ & - \\
\hline & Glucose-6-phosphate & - & $3.15 \uparrow$ \\
\hline \multirow{3}{*}{ 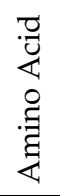 } & Aspartic Acid & $2.8 \uparrow$ & - \\
\hline & Lysine & $4.13 \uparrow$ & - \\
\hline & Tryptophane & $1.87 \uparrow$ & $2.89 \uparrow$ \\
\hline \multirow{2}{*}{ 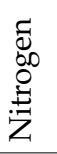 } & Pyroglutamic Acid & $1.65 \uparrow$ & - \\
\hline & Glutamic acid & $2.37 \uparrow$ & - \\
\hline \multirow{3}{*}{$\underset{\stackrel{\Xi}{\Xi}}{\stackrel{\Xi}{Z}}$} & Phenylethylamine & $3.52 \uparrow$ & $4.39 \uparrow$ \\
\hline & 2-amino-1-phenylethanol & $12.83 \uparrow$ & $1.47 \uparrow$ \\
\hline & Tyramine & $1.68 \uparrow$ & $2.51 \uparrow$ \\
\hline \multirow{2}{*}{ 范 } & Glycerol-phosphate & - & $1.79 \uparrow$ \\
\hline & Beta-glycerolphosphate & - & $1.65 \uparrow$ \\
\hline \multirow{4}{*}{ 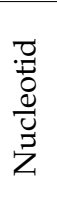 } & Uracil & $6.11 \uparrow$ & $11.01 \uparrow$ \\
\hline & Uridine 5'-monophosphate & $0.41 \downarrow$ & $3.81 \uparrow$ \\
\hline & Ribulose-5-phosphate & $1.38 \uparrow$ & $9.27 \uparrow$ \\
\hline & Pyrophosphate & $1.49 \uparrow$ & $5.89 \uparrow$ \\
\hline
\end{tabular}

The multivariate statistical analysis of GC-MS metabolomics results was performed using PCA (Supplement Figure S1) and PLS-DA methods. PLS-DA analysis was performed to further investigate the altered metabolite profile in cells when ciprofloxacin was administered in combination with curcumin, cinnamaldehyde, baicalein, and esculin hydrate, respectively, compared with ciprofloxacin alone (Table 2). Firstly, the statistical goodness and robustness of the models were evaluated using R2 (the fraction of variance explained by a component) and Q2 (the fraction of the total variation predicted by a component) values, respectively. The R2 and Q2 values for the models were higher than 0.9 for both, indicating the validity of the methods and showing that the models were stable and used the settings for the variables to calculate the predictions. The scores plots showed that the metabolomics profile of ciprofloxacin and ciprofloxacin plus curcumin, cinnamaldehyde, baicalein, and esculetin hydrate were significantly different from each other (Figure 4A,C,E,G). The VIP graphs are shown in Figure 4B,D,F,G.

\section{Discussion}

A decrease in tolerance to antimicrobials can be observed in P. aeruginosa cells living in biofilms compared with planktonic forms. Natural compounds such as cinnamaldehyde, curcumin, esculetin hydrate, and baicalein can be considered as alternative antimicrobial 
agents that are not affected by drug resistance developed by bacteria. The inhibition of the QS network is a potential strategy for controlling infections caused by P. aeruginosa. The impacts of various natural and synthetic compounds on the QS system of bacteria have been investigated [14-16]. Investigating the effects of sub-MIC concentrations of agents that can be alternatives to antibiotics for biofilm formation is particularly important for combating both drug resistance and biofilm-related infections, as they are less likely to apply selective pressure for resistance development [6]. In recent years, anti-biofilm effect studies involving drug combinations have shown that the effects of the combinations are higher than for the single agents $[17,18]$. It was reported in a study that $S$. aureus and $C$. albicans were resistant to curcumin and imidazole both in mono and dual biofilms, but when these agents were used together, there was a significant decrease in the number of living cells in the biofilms [18]. In the study by Bahari et al., it was shown that the combination of azithromycin and gentamicin with curcumin significantly reduced the MIC values of the antibiotics, the clumping and twitching motility of P. aeruginosa, and the formation of biofilms [15]. Sub-MIC concentrations of cinnamaldehyde were shown to be able to decrease the formation of biofilms by S. epidermidis, MRSA, and E. coli $[14,19,20]$. The antibacterial activity of catechin hydrate, a flavonoid found in tea, wine, some fruits, vegetables, and chocolate, against $S$. aureus clinical strains and the synergistic effect of catechin hydrate with erythromycin and clindamycin were reported in a study [21]. In addition, it has been found to inhibit the biosynthesis of QS-associated virulence factors in P. aeruginosa [22].

In our study, we determined the MBIC and MBEC values by examining the anti-biofilm effect of 10 potential QS inhibitor compounds and ciprofloxacin against $P$. aeruginosa biofilm cells, both alone and in combination. Similar to the literature, our results showed that ciprofloxacin and compound combinations were effective at inhibiting P. aeruginosa at lower concentrations compared with ciprofloxacin alone. No effect for the combinations on reducing the MBEC of ciprofloxacin was observed, although the most reductions in the MBICs of ciprofloxacin were determined when in combination with curcumin, baicalein, esculetin hydrate, and cinnamaldehyde. These combinations with low MBICs may be significant in the treatment of biofilm-associated P. aeruginosa infections.

Ciprofloxacin and the compounds alone showed an inhibitory effect on both the motility and pyocyanin synthesis of P. aeruginosa. However, except for the effects of combinations of ciprofloxacin with curcumin and esculetin hydrate on the cells' twitching activity, a synergistic effect increasing the inhibition was not observed for the other combinations.

Antimicrobial treatment efficacy was less successful in dual- or multiple-species biofilms in vitro than biofilms alone [23-25]. As biofilm infections are caused by microbial cells consisting of more than one species in the clinic, we compared the effects of the agent combinations both in P. aeruginosa alone and in triple-species biofilms. Azathioprine in combination with ciprofloxacin was determined to be more effective at killing P. aeruginosa in mono-species biofilms compared with the triple-species biofilm formed by P. aeruginosa, S. aureus, and E. facealis. However, contrasting results were obtained for combinations of ciprofloxacin with curcumin, catechin hydrate, baicalein, L-canavanine, esculin hydrate, and cinnamaldehyde. These results show that the presence of a polymicrobial biofilm environment should also be taken into account for treatment strategies.

Although it has been reported in the literature that some combinations tested in our study provide an antibiofilm effect, there are very few studies explaining these mechanisms [26]. In the presented study, cellular metabolomes of $P$. aeruginosa treated with sub-MBIC concentrations of both ciprofloxacin and combinations of ciprofloxacin with curcumin, baicalein, cinnamaldehyde, and esculetin hydrate were analyzed to clarify the mechanisms of action for the compounds. We observed that uracil ( -7.7 and -5.9 -fold in combination with curcumin and cinnamaldehyde, respectively) and UMP metabolites (-50-fold in combination with cinnamaldehyde) involved in pyrimidine synthesis were significantly reduced in cells treated with curcumin and cinnamaldehyde combinations compared with ciprofloxacin alone. Pyrimidine synthesis is a complex pathway that begins 
with the transformation of L-glutamate to uridine monophosphate (UMP) for RNA and DNA production [27,28]. Pyrimidine synthesis plays a significant role in the regulation of many cell functions of P. aeruginosa [29]. In phenotypic studies, uracil is found to be effective on elastase, rhamnolipid synthesis, pseudomonas quinolone signal and swarming action, which are among the QS-related virulence factors. Whole transcriptome analysis of the UMP synthesis changed mutant strains showed that hundreds of QS-related genes and biofilm formation were suppressed and inhibited. However, the addition of uracil to the medium provided the re-expression of these genes and biofilm formation [30].

The down-regulated QS gene results obtained for curcumin and cinnamaldehyde, which provide a synergistic effect to ciprofloxacin in killing biofilm cells, and decreased uracil and UMP levels, support studies expressing the link between pyrimidine synthesis and QS inhibition in P. aeruginosa. In contrast to curcumin and cinnamaldehyde, the uracil and UMP metabolite levels and the upregulation of all genes increased in biofilm cells treated with combinations of ciprofloxacin with baicalein and esculin hydrate. These results show that one of the multifactorial mechanisms that can cause QS inhibition by curcumin and cinnamaldehyde is the uracil and UMP mediated pyrimidine mechanism.

Alginate is a capsular polysaccharide that has a significant role in P. aeruginosa virulence by helping recover from the effects of antipseudomonal drugs and the host immune system [31]. Ahmar et al. (2019) demonstrated a direct relationship between the UMP and UTP pyrimidine concentration and the mucoidity of $P$. aeruginosa at the level of transcriptional regulation mediated by the PalgD activity [27]. Similarly, we found the decreased uracil and UMP concentration and alginate gene expression levels in cells exposed to combinations of ciprofloxacin with curcumin and cinnamaldehyde, whereas increased levels of uracil and UMP concentration and gene expression in cells exposed to combinations of ciprofloxacin with baicalein and esculin hydrate were found. Therefore, another possible mechanism of killing biofilm cells with curcumin and cinnamaldehyde may be the decrement in alginate synthesis, which is indirectly associated with the decrease in pyrimidine metabolite levels.

Lipopolysaccharide (LPS) is a complex glycolipid composed of lipid A, core o-saccharide and hypervariable long-chain o-polysaccharide and plays important roles in the integrity of the outer membrane permeability barrier of $P$. aeruginosa [32]. Phosphoglycerate ( $25-$ fold), cytidine-monophosphate ( -2.85 -fold), glycine $(-2.5$-fold), and ribulose-5-phosphate (-4.3-fold) metabolites, detected in lower amounts in cells exposed to a combination of ciprofloxacin with curcumin, play an important role in different stages of LPS synthesis.

Biogenic amines (BAs) regulate chromosomal and ribosomal organization; DNA, RNA, and protein synthesis in bacteria; and protect bacteria from osmotic stress. They are also known to regulate the swarming action and biofilm formation of P. aeruginosa [33,34]. Unlike those exposed to combinations of ciprofloxacin with baicalin and esculin hydrate, cells treated with combinations of ciprofloxacin with curcumin and cinnamaldehyde had a low level of 2-phenyl-ethylamine, 2-amino-1-phenyl ethanol, and tyramine. The decrease in BA levels in the cells treated with combinations with curcumin and cinnamaldehyde could be another reason for $P$. aeruginosa biofilm inhibition.

In conclusion, our multifactorial results, such as uracil and UMP-mediated pyrimidine synthesis, LPS biosynthesis, alginate production, and QS systems, indicate the reasons for curcumin and cinnamaldehyde-mediated P. aeruginosa biofilm inhibition. Curcumin, cinnamaldehyde, baicalein, and esculin reduced the inhibitory concentration of ciprofloxacin against biofilm cells by up to 64-fold. Using these compounds together with ciprofloxacin will help achieve successful results in the fight against ciprofloxacin-resistant $P$. aeruginosa biofilm infections.

Supplementary Materials: The following are available online at https: / www.mdpi.com/article/ 10.3390/antibiotics10040414/s1, Table S1. Metabolites and relative amounts identified by GC-MS analysis. Metabolites that differ between groups are written in bold. ${ }^{*}: p<0.05$ indicates that the difference is statistically significant. ${ }^{* *}$ : Data are values after normalization MSTUS and mean centering. Table S2: Primers for P. aeruginosa for RT-qPCR. ( ${ }^{*}$ reference genes), Figure S1. Scanning electron 
micrographs of single (P. aeruginosa) and triple-species biofilms formed by P. aeruginosa, S. aureus and E. faecalis. Panel A: P. aeruginosa, magnification $5.000 \times$, Panel B: P. aeruginosa, magnification $20.000 \times$, Panel C: Triple-species biofilm, magnification 5.000 $\times$, Panel D: Triple-species biofilm, magnification $20.000 \times$. S.a: Staphylococcus aureus, P.a: Pseudomonas aeruginosa, E.f: Enterococcus faecalis. Figure S2: Principal component analysis (PCA) score plots of metabolic profiles in P. aeruginosa biofilms. Cip: Ciprofloxacin, Cip + Bai: Ciprofloxacin and Baicalein, Cip + Cur: Ciprofloxacin and Curcumin, Cip + Esc: Ciprofloxacin and Esculetin hydrate, Cip + Cal: Ciprofloxacin and Cinnamaldehyde.

Author Contributions: Conceptualization, D.K. and M.S.; formal analysis, D.K., T.R., and E.N.; investigation, D.K., T.R., and E.N.; methodology, D.K., T.R., and E.N.; project administration, D.K.; software, E.N.; supervision, D.K. and M.S.; validation, D.K., T.R., and E.N.; writing-original draft, D.K., T.R., and E.N.; writing-review and editing, D.K., T.R., E.N., and M.S. All authors have read and agreed to the published version of the manuscript.

Funding: This work was supported by grants from the Scientific and Technological Research Council of Turkey (grant number 115S550).

Institutional Review Board Statement: Not applicable.

Informed Consent Statement: Not applicable.

Data Availability Statement: The data generated is contained within the article.

Conflicts of Interest: The authors have no conflict of interest to declare.

\section{References}

1. Tuon, F.F.; Gortz, L.W.; Rocha, J.L. Risk factors for pan-resistant Pseudomonas aeruginosa bacteremia and the adequacy of antibiotic therapy. Braz. J. Infect. Dis. 2012, 16, 351-356. [CrossRef] [PubMed]

2. Yang, L.; Liu, Y.; Wu, H.; Hoiby, N.; Molin, S.; Song, Z.J. Current understanding of multi-species biofilms. Int. J. Oral Sci. 2011, 3, 74-81. [CrossRef] [PubMed]

3. Bayramov, D.F.; Neff, J.A. Beyond conventional antibiotics-New directions for combination products to combat biofilm. Adv. Drug Deliver Rev. 2017, 112, 48-60. [CrossRef]

4. Bassler, B.L. How bacteria talk to each other: Regulation of gene expression by quorum sensing. Curr. Opin. Microbiol. 1999, 2, 582-587. [CrossRef]

5. Starkey, M.; Lepine, F.; Maura, D.; Bandyopadhaya, A.; Lesic, B.; He, J.X.; Kitao, T.; Righi, V.; Mi-lot, S.; Tzika, A.; et al. Identification of Anti-virulence Compounds That Disrupt Quorum-Sensing Regulated Acute and Persistent Pathogenicity. PLoS Pathog. 2014, 10, e1004321. [CrossRef] [PubMed]

6. Brackman, G.; Hillaert, U.; Van Calenbergh, S.; Nelis, H.J.; Coenye, T. Use of quorum sensing inhibitors to interfere with biofilm formation and development in Burkholderia multivorans and Burkholderia cenocepacia. Res. Microbiol. 2009, 160, 144-151. [CrossRef] [PubMed]

7. Fazzini, R.A.B.; Skindersoe, M.E.; Bielecki, P.; Puchalka, J.; Givskov, M.; dos Santos, V.A.P.M. Protoanemonin: A natural quorum sensing inhibitor that selectively activates iron starvation response. Environ. Microbiol. 2013, 15, 111-120. [CrossRef] [PubMed]

8. Ulrey, R.K.; Barksdale, S.M.; Zhou, W.D.; van Hoek, M.L. Cranberry proanthocyanidins have anti-biofilm properties against Pseudomonas aeruginosa. BMC Complem. Altern. Med. 2014, 14, 1-12. [CrossRef] [PubMed]

9. Andrews, J.M. Determination of minimum inhibitory concentrations. J. Antimicrob. Chemother. 2001, 48, 5-16. [CrossRef]

10. Kart, D.; Tavernier, S.; Van Acker, H.; Nelis, H.J.; Coenye, T. Activity of disinfectants against multi-species biofilms formed by Staphylococcus aureus, Candida albicans and Pseudomonas aeruginosa. Biofouling 2014, 30, 377-383. [CrossRef]

11. Kart, D.; Ciftci, S.Y.; Nemutlu, E. Altered metabolomic profile of dual-species biofilm: Interactions between Proteus mirabilis and Candida albicans. Microbiol. Res. 2020, 230, 126346. [CrossRef]

12. Reimmann, C.; Beyeler, M.; Latifi, A.; Winteler, H.; Foglino, M.; Lazdunski, A.; Haas, D. The global activator GacA of Pseudomonas aeruginosa PAO positively controls the production of the autoinducer N-butyryl-homoserine lactone and the formation of the virulence factors pyocyanin, cyanide, and lipase. Mol. Microbiol. 1997, 24, 309-319. [CrossRef]

13. Cankirili, N.K.; Kart, D.; Celebi-Saltik, B. Evaluation of the biofilm formation of Staphylococcus aureus and Pseudomonas aeruginosa on human umbilical cord CD146+stem cells and stem cell-based decellularized matrix. Cell Tissue Bank. 2020, 21, $215-231$. [CrossRef]

14. Albano, M.; Crulhas, B.P.; Alves, F.C.B.; Pereira, A.F.M.; Andrade, B.F.M.T.; Barbosa, L.N.; Furlanetto, A.; Lyra, L.P.D.; Rall, V.L.M.; Fernandes, A. Antibacterial and anti-biofilm activities of cinnamaldehyde against S. epidermidis. Microb. Pathog. 2019, 126, 231-238. [CrossRef]

15. Bahari, S.; Zeighami, H.; Mirshahabi, H.; Roudashti, S.; Haghi, F. Inhibition of Pseudomonas aeruginosa quorum sensing by subinhibitory concentrations of curcumin with gentamicin and azithromycin. J. Glob. Antimicrob. Resist. 2017, 10, 21-28. [CrossRef] [PubMed] 
16. El-Mowafy, S.A.; Abd El Galil, K.H.; El-Messery, S.M.; Shaaban, M.I. Aspirin is an efficient inhibitor of quorum sensing, virulence and toxins in Pseudomonas aeruginosa. Microb. Pathog. 2014, 74, 25-32. [CrossRef] [PubMed]

17. Bag, A.; Chattopadhyay, R.R. Synergistic antibiofilm efficacy of a gallotannin 1,2,6-tri-O-galloyl-beta-D-glucopyranose from Terminalia chebula fruit in combination with gentamicin and trime-thoprim against multidrug resistant uropathogenic Escherichia coli biofilms. PLoS ONE 2017, 12, e0178712. [CrossRef] [PubMed]

18. Tan, Y.L.; Leonhard, M.; Moser, D.; Ma, S.; Schneider-Stickler, B. Antibiofilm efficacy of curcumin in combination with 2aminobenzimidazole against single- and mixed-species biofilms of Candida albicans and Staphylococcus aureus. Colloid Surf. B 2019, 174, 28-34. [CrossRef] [PubMed]

19. Albano, M.; Alves, F.C.; Andrade, B.F.; Barbosa, L.N.; Pereira, A.F.; de Souza, M.D.; Rall, V.L.; Júnior, A.F. Antibacterial and anti-staphylococcal enterotoxin ac-tivities of phenolic compounds. Innov. Food Sci. Emerg. 2016, 38, 83-90. [CrossRef]

20. Borges, A.; Lopez-Romero, J.C.; Oliveira, D.; Giaouris, E.; Simoes, M. Prevention, removal and inac-tivation of Escherichia coli and Staphylococcus aureus biofilms using selected monoterpenes of essential oils. J. Appl. Microbiol. 2017, 123, 104-115. [CrossRef]

21. Miklasinska, M.; Kepa, M.; Wojtyczka, R.D.; Idzik, D.; Dziedzic, A.; Wasik, T.J. Catechin Hydrate Augments the Antibacterial Action of Selected Antibiotics against Staphylococcus aureus Clinical Strains. Molecules 2016, 21, 244. [CrossRef]

22. Vandeputte, O.M.; Kiendrebeogo, M.; Rajaonson, S.; Diallo, B.; Mol, A.; El Jaziri, M.; Baucher, M. Identification of Catechin as One of the Flavonoids from Combretum albiflorum Bark Extract That Reduces the Production of Quorum-Sensing-Controlled Virulence Factors in Pseudomonas aeruginosa PAO1. Appl. Environ. Microb. 2010, 76, 243-253. [CrossRef]

23. Wang, X.; Li, X.; Ling, J. Streptococcus gordonii LuxS/autoinducer-2 quorum-sensing system modulates the dual-species biofilm formation with Streptococcus mutans. J. Basic Microbiol. 2017, 57, 605-616. [CrossRef]

24. Peters, B.M.; Ward, R.M.; Rane, H.S.; Lee, S.A.; Noverr, M.C. Efficacy of ethanol against Candida albicans and Staphylococcus aureus polymicrobial biofilms. Antimicrob. Agents Chemother. 2013, 57, 74-82. [CrossRef]

25. Wu, C.Y.; Su, T.Y.; Wang, M.Y.; Yang, S.F.; Mar, K.; Hung, S.L. Inhibitory effects of tea catechin epigallocatechin-3-gallate against biofilms formed from Streptococcus mutans and a probiotic lactobacillus strain. Arch. Oral Biol. 2018, 94, 69-77. [CrossRef]

26. Tan, Y.L.; Ma, S.; Leonhard, M.; Moser, D.; Haselmann, G.M.; Wang, J.; Eder, D.; Schneider-Stickler, B. Enhancing antibiofilm activity with functional chitosan nanoparticles targeting biofilm cells and biofilm matrix. Carbohyd. Polym. 2018, $200,35-42$. [CrossRef]

27. Al Ahmar, R.; Kirby, B.D.; Hongwei, D.Y. Pyrimidine Biosynthesis Regulates the Small-Colony Variant and Mucoidy in Pseudomonas aeruginosa through Sigma Factor Competition. J. Bacteriol. 2019, 201. [CrossRef]

28. Odonovan, G.A.; Neuhard, J. Pyrimidine Metabolism in Microorganisms. Bacteriol. Rev. 1970, 34, 278. [CrossRef]

29. Beaume, M.; Kohler, T.; Fontana, T.; Tognon, M.; Renzoni, A.; van Delden, C. Metabolic pathways of Pseudomonas aeruginosa involved in competition with respiratory bacterial pathogens. Front. Microbiol. 2015, 6, 321. [CrossRef] [PubMed]

30. Ueda, A.; Attila, C.; Whiteley, M.; Wood, T.K. Uracil influences quorum sensing and biofilm formation in Pseudomonas aeruginosa and fluorouracil is an antagonist. Microb. Biotechnol. 2009, 2, 62-74. [CrossRef]

31. Ramsey, D.M.; Wozniak, D.J. Understanding the control of Pseudomonas aeruginosa alginate synthesis and the prospects for management of chronic infections in cystic fibrosis. Mol. Microbiol. 2005, 56, 309-322. [CrossRef] [PubMed]

32. Whitfield, C.; Trent, M.S. Biosynthesis and Export of Bacterial Lipopolysaccharides. Annu. Rev. Biochem. 2014, 83, 99-128. [CrossRef] [PubMed]

33. Luengo, J.M.; Olivera, E.R. Catabolism of biogenic amines in Pseudomonas species. Environ. Microbiol. 2020, 22, 1174-1192. [CrossRef] [PubMed]

34. Shah, P.; Swiatlo, E. A multifaceted role for polyamines in bacterial pathogens. Mol. Microbiol. 2008, 68, 4-16. [CrossRef] [PubMed] 